\title{
生物合成高能化学物质
}

陈子鹏 1 , 赵钰沁 2 , 王宸 ${ }^{1}$, 王秀秀 1 , 魏炜 2 , 赵劲 1 , ${ }^{*}$

1 南京大学化学化工学院, 南京 210023

2 南京大学生命科学学院, 南京 210023

摘要: 从分子到细胞, 从个体到社会, 能量的需求和转化无处不在。探索高能物质的合成与利用, 具有较重要的科 学意义和应用价值。目前高能物质的化学合成通常污染较大、耗能较高, 且往往副产物较多, 难以分离纯化。相较 于传统的化学合成方法, 生物合成具有绿色环保和高效利用清洁能源等特点, 是符合国家 “碳中和” 目标的新型合 成路径。高能物质的生物合成, 将是未来的迅速发展方向之一。本文将从能源物质、含能材料和天然高能物质三个 方面，选取四种代表性高能物质，介绍其生物合成途径及与之相关的关键金属酶。

关键词: 高能物质; 生物合成; 氢气; 氧化偶氮键; 丁三醇; 多聚磷酸盐

中图分类号: G64; O6

\section{Biosynthesis of High-Energy Chemicals}

\author{
Zipeng Chen ${ }^{1}$, Yuqin Zhao ${ }^{2}$, Chen Wang ${ }^{1}$, Xiuxiu Wang ${ }^{1}$, Wei Wei ${ }^{2}$, Jing Zhao ${ }^{1,}{ }^{*}$ \\ ${ }^{1}$ School of Chemistry and Chemical Engineering, Nanjing University, Nanjing 210023, China. \\ ${ }^{2}$ School of Life Sciences, Nanjing University, Nanjing 210023, China.
}

\begin{abstract}
From molecules to cells, the energy demand is ubiquitous in our society. Synthesis and utilization of highenergy chemicals is a major challenge scientifically and economically. The current chemical synthesis of high-energy compounds often generates pollution and consumes a lot of energy. With the existence of by-products, it's hard to separate target products. Compared to chemical synthesis, biosynthesis is considered environmentally friendly and cost-effective energy, and is in accord with the Carbon Neutralization goal. Biosynthesis of high-energy chemicals is one of the fastest growing fields. This article will choose 4 chemicals, in terms of energy source, energetic materials and bio-high-energy compounds, to briefly introduce the correspondent biosynthesis pathway and key metalloenzyme.
\end{abstract}

Key Words: High-energy chemicals; Biosynthesis; Hydrogen; Azoxy bond; Butane-1,2,4-triol; Polyphosphate

2020年9月, 习近平总书记在第75届联合国大会一般性辩论上提出, 中国力争 2030 年实现碳达 峰, 并努力争取2060年前实现碳中和 ${ }^{[1]}, 2021$ 年的政府工作报告中, 这一重大战略决策再度被提 及 ${ }^{[2]}$ 。对于实现 “碳中和” 目标, 生物合成技术的发展与应用起到不可或缺的作用 ${ }^{[3]}$, 与化学合成相 比, 生物合成可以实现从原料源头上降低碳排放。生物合成依赖于各种酶, 酶催化的反应转化率高、 反应条件温和, 并且生成产物的结构专一 ${ }^{[4]}$, 可以实现较好的原子经济性, 并降低能耗 ${ }^{[5]}$ 。目前, 世 界上主要的能源供应来源于化石燃料, 其他高能物质的生产也依赖于化学合成, 污染高, 碳排放量

收稿: 2021-10-08; 录用: 2021-11-15; 网络发表: 2021-12-22

“通讯作者, Email: jingzhao@nju.edu.cn

基金资助: 国家自然科学基金杰出青年科学基金(22025701); 国家自然科学基金面上项目(22177048); 国家重点研发计划合成生物学专项子课题 (2019YFA0905801) 
大。然而, 研究人员发现, 自然界中存在可以合成能源物质、含能材料的生物, 并已经破解出相当 一部分生物合成途径 ${ }^{[6,7]}$, 这将为人们更好地应用生物合成技术实现节能减排指明方向。

本文将从能源物质、含能材料和天然高能物质三个方面入手, 选取氢气、丁三醇、氧化偶氮化 合物和多聚磷酸盐几种代表性高能物质, 分析讨论它们的生物合成途径以及与之相关的关键金属酶。

\section{1 能源物质的生物合成一一氢气}

能源物质中, 氢气是当之无愧的清洁能源, 但基于裂解水的制氢技术耗能较高, 并且设备容易 腐蚀, 基于石油/煤化工的制氢技术碳排放高 ${ }^{[8]}$, 幸运的是, 自然界中存在具有产氢能力的氢化酶 (Hydrogenase), 对这类酶的研究为制氢工业开辟了一条新的道路。

氢化酶是金属酶, 根据其金属离子的不同可分为 $[\mathrm{Fe}]$ 氢化酶、 $[\mathrm{NiFe}]$ 氢化酶和 $[\mathrm{FeFe}]$ 氢化酶三 种 ${ }^{[9]}$ 。[Fe]氢化酶是一种不含铁硫簇的氢化酶, 具有 FeGP辅因子, 这种酶存在于产甲烷菌中, 可逆地 催化methenyltetrahydromethanopterin $\left(\right.$ methenyl- $\mathrm{H}_{4} \mathrm{MPT}^{+}$)与氢气反应, 产生methenyl- $\mathrm{H}_{4} \mathrm{MPT}$ 和一个质 子(图1) ${ }^{[10-13]}$, Methenyl- $\mathrm{H}_{4} \mathrm{MPT}^{+}$是产甲烷菌的一个甲基转移中间体, 这一反应是产甲烷菌中将 $\mathrm{CO}_{2}$ 还原成 $\mathrm{CH}_{4}$ 代谢通路的中间环节, 该反应的顺利进行维系了产甲烷菌的能量代谢过程。

(a)

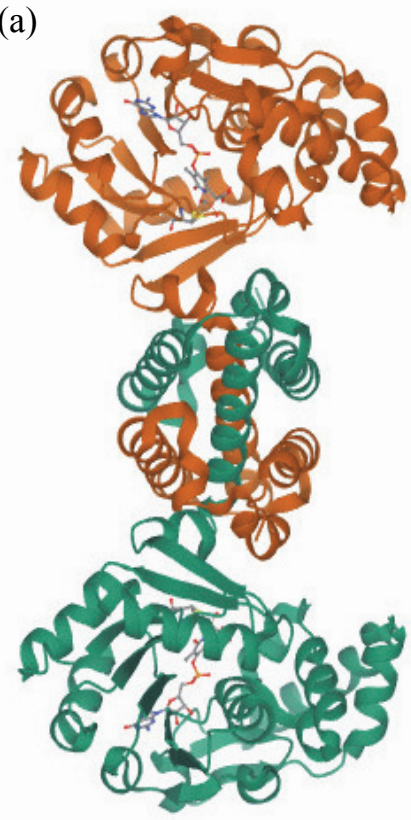

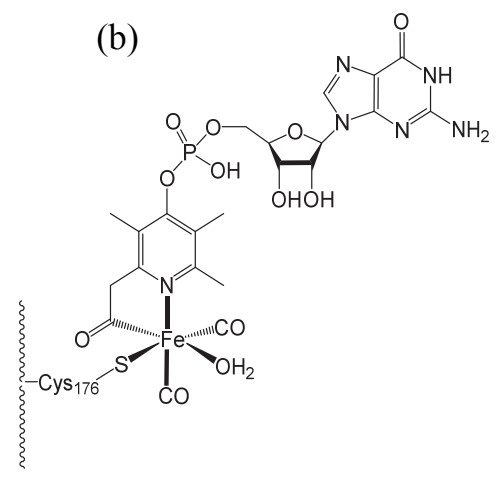

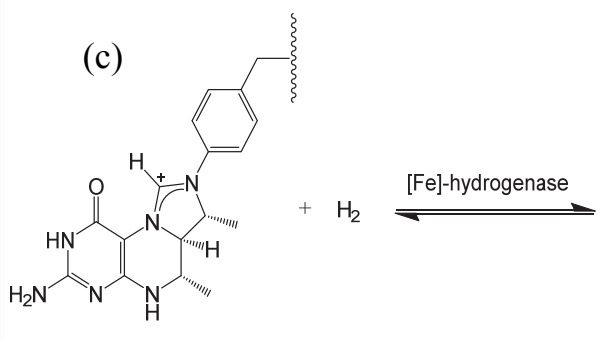

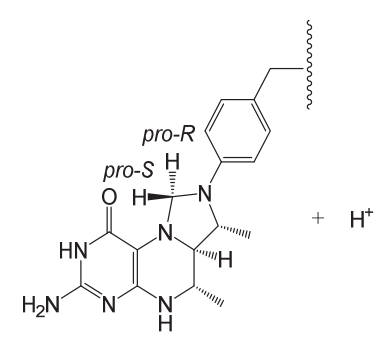

图1 (a) [Fe]氢化酶的晶体结构(PDBID: 3DAG) ${ }^{[10] ;}$ (b) FeGP辅因子; (c) [Fe]氢化酶所催化的可逆产氢反应 ${ }^{[13]}$

$[\mathrm{FeFe}]$ 氢化酶和 $[\mathrm{NiFe}]$ 氢化酶具有铁硫簇, $[\mathrm{FeFe}]$ 氢化酶还具有 $\mathrm{H}$ 簇(图 $2 \mathrm{a})^{[14,15]}[\mathrm{FeFe}]$ 氢化酶存 在于厌氧菌中, 目前Stripp课题组 ${ }^{[16]}$ 已阐明其产氢的质子传递机理, 即当缺乏天然发色团的高活性铁 硫酶经过光还原后, 活性位点辅助因子(H簇)的碱度增强, 引发了不连续质子转移(图 $2 \mathrm{~b}$ )。

$[\mathrm{NiFe}]$ 氢化酶存在于细菌和古菌中, 这种氢化酶更偏向于将氢气裂解为质子与氢负离子 $[17,18]$ 。大 肠杆菌中也存在 $[\mathrm{NiFe}]$ 氢化酶, 这种酶属于FHL复合体, 是大肠杆菌厌氧发酵物产生氢气的来源 (图3) ${ }^{[19]}$ 。

在氢化酶产氢的研究实践中, 研究人员通常将氢化酶与无机催化剂联用, 构建生物-无机杂化催 化体系(Bio-inorganic hybrid catalytic system), 从而提高酶的产氢效率 ${ }^{[20]}$ 。由于半导体具有本多-藤岛 效应 ${ }^{[21]}$, 研究人员常将半导体与氢化酶联用, 构建半人工光合体系, 例如Honda团队 ${ }^{[22]}$ 通过在大肠 杆菌中表达 $[\mathrm{FeFe}]$ 氢化酶同时外加纳米 $\mathrm{TiO}_{2}$ 实现了产氢, 浙江大学唐睿康团队 ${ }^{[23]}$ 以绿藻为产氢底盘 

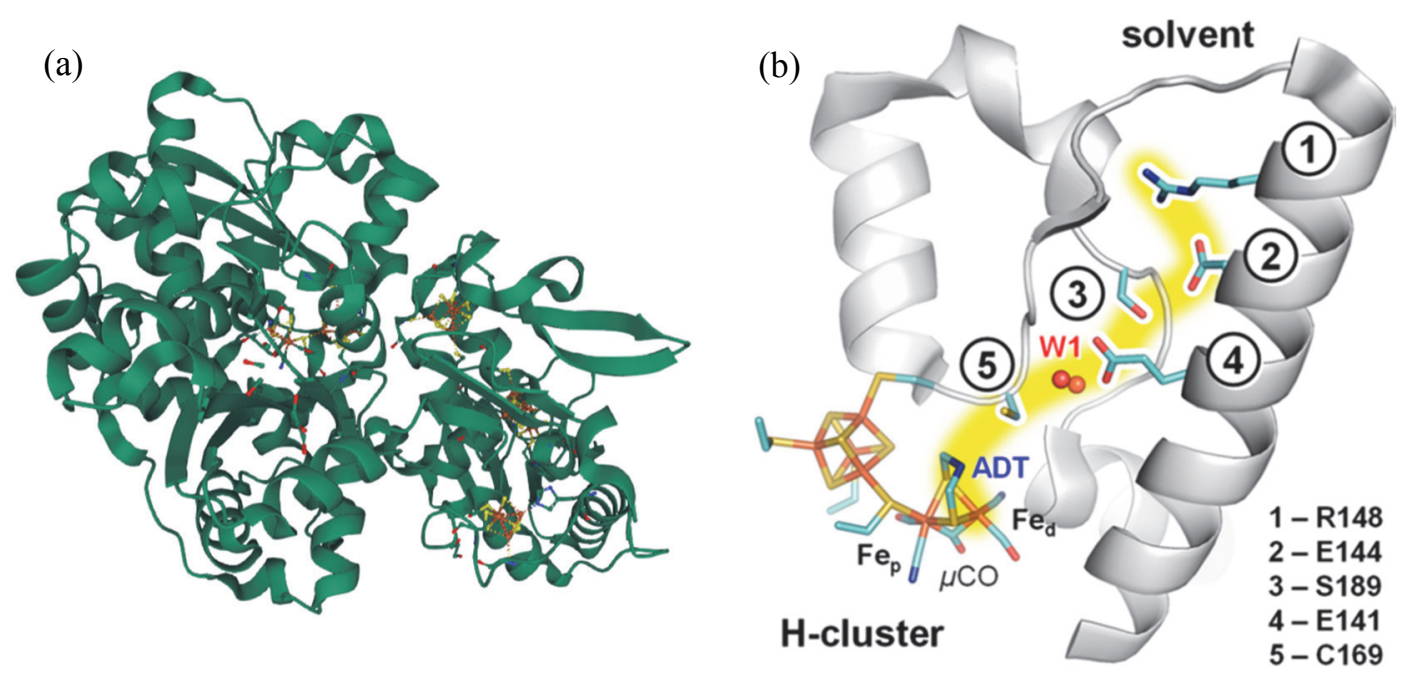

图2 (a) [FeFe]氢化酶的结构(PDBID: 3C8Y) ${ }^{[15]}$; (b) [FeFe]氢化酶中与质子迁移相关的氨基酸残基与辅基 ${ }^{[16]}$

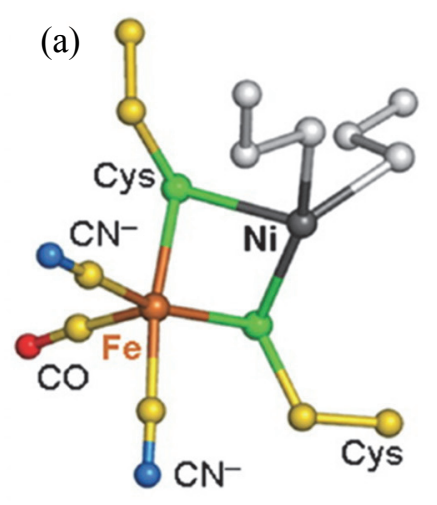

(b) Periplasm

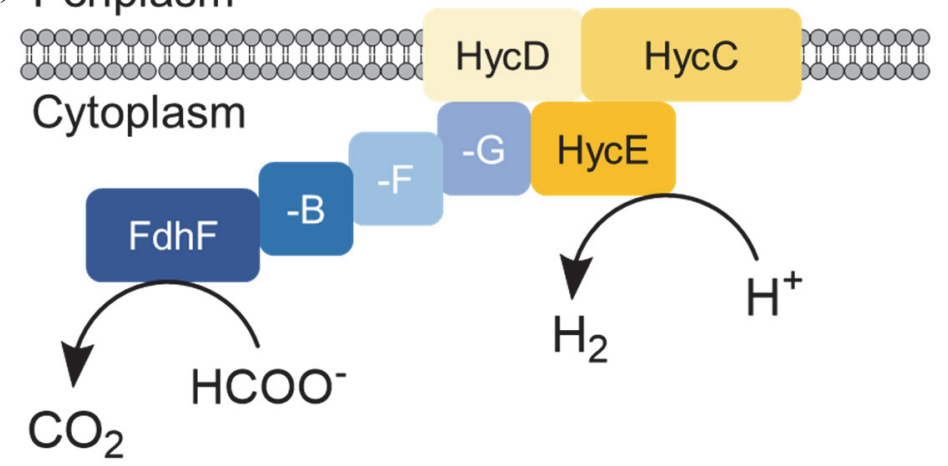

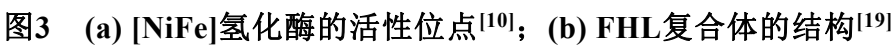

生物, 通过向绿藻中加入无定形 $\mathrm{SiO}_{2}$ 引起绿藻硅矿化聚集成生物小球, 并加入 $\mathrm{WO}_{3}$ 引发氢气产生, 南京大学赵劲和魏炜团队 ${ }^{[24]}$ 通过在大肠杆菌表面展示PbrR金属蛋白使之原位合成CdS半导体量子点 并同时表达氢化酶Hya, 从而实现大肠杆菌光催化产氢。上海交通大学㚞春海院士团队 ${ }^{[25]}$ 通过外加 $\mathrm{Mg}(\mathrm{OH})_{2}$ 诱导绿藻聚集, 实现长期连续产氢。电催化是制氢的常用策略, 也有研究人员将电极或电 催化剂与氢化酶联用, 促进产氢 ${ }^{[26,27]}$ 。

同时, 研究人员也不断尝试对产氢酶进行人工改造, 或尝试模拟氢化酶的结构设计新型产氢催 化剂。潘惠杰等 ${ }^{[28]}$ 模拟 $[\mathrm{Fe}]$ 氢化酶的结构, 设计了一种新型 $\mathrm{Mn}$ 配合物催化剂, 吴骊珠院士团队 ${ }^{[29]}$ 模 仿 $[\mathrm{NiFe}]$ 氢化酶的活性中心, 构建了一种 $\mathrm{NiRu}-\mathrm{RuNi}$ 二聚体金属簇催化剂。Armstrong团队 ${ }^{[30]}$ 通过将 $[\mathrm{NiFe}]$ 氢化酶口袋中的半胱氨酸替换成硒代半胱氨酸, 大大提升了氢化酶的催化效率和氧气耐受能 力。

随着生物产氢研究的不断深入, 人们对氢化酶的理解不断加深, 将极大促进生物产氢从实验室 走向工业界的转变。特别是生物-无机杂化催化体系的应用, 将推动产氢技术不断向低能耗与低污染 前进。

\section{2 含能材料的生物合成- $1,2,4-丁 三$ 醇和氧化偶氮化合物}

含能材料(Energetic materials)是指一种或多种化合物, 具有易燃或易爆官能团, 会剧烈氧化释放 
大量能量和远超自身体积的气体 ${ }^{[31]}$ 。含能材料在民间及军事上存在很多用途, 但其生产主要依赖化 学合成 ${ }^{[32]}$, 如著名的梯恩梯炸药(三硝基甲苯, TNT)、黑索金炸药(环三亚甲基三硝胺, RDX)和奥托 金炸药(环四亚甲基四硝胺, HMX)的生产, 需要消耗大量硫酸、硝酸对底物进行硝化, 生产过程的 危险性较高。近年来随着生物合成技术的发展, 含能材料的生物合成也正在启动。

\section{1 丁三醇及衍生物}

1,2,4-丁三醇(Butane-1,2,4-triol, BT) 是一种具有极高价值的有机合成中间体, 尤其是其可以作为 高能燃料和炸药的前体物质。其硝化产物 1,2,4-丁基三硝酸酯(BTTN)具有低温力学性、冲击稳定性和 使用安全性等特点, 在武器固体推进剂和发射药配方中有着极其重要的作用, 被视为传统硝酸甘油

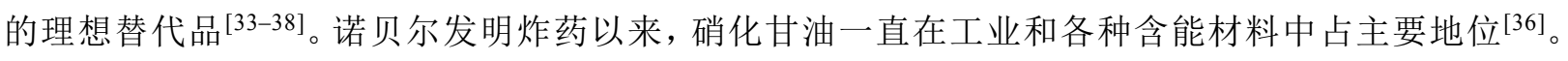
因此，为了实现这一 “替代”，BT得到了国内外科研工作者的普遍关注。

目前 BT的工业化生产主要采用 $\mathrm{NaBH}_{4}$ 还原苹果酸二酯、 $\mathrm{Rb} / \mathrm{C}$ 催化苹果酸加氢、马来酸二乙酯 $/ \mathrm{Cu} / \mathrm{Cr}$ 催化加氢等化学合成 ${ }^{[39,40]}$ 。这些方法的工艺条件苛刻, 且采用的高压催化加氢等相对危险, 合 成过程中涉及到的录盐和大量酸性溶剂会对环境有极大的污染。反应条件温和、安全系数高、对环 境友好的生物合成法成为当下的研究热点。

自然界中暂时还未发现天然的BT生物合成途径 ${ }^{[36]}$, 但通过合成生物学方法对天然微生物进行改 造, BT的生物合成得以实现。2003年, Frost团队首次报道利用假单胞杆菌和大肠杆菌两种微生物, 以木糖和阿拉伯糖为底物, 经四步酶促反应合成BT ${ }^{[36]}$ (图4)。

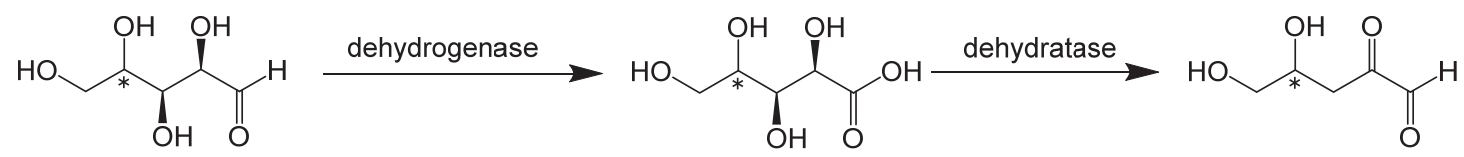

D-xylose/L-arabinose

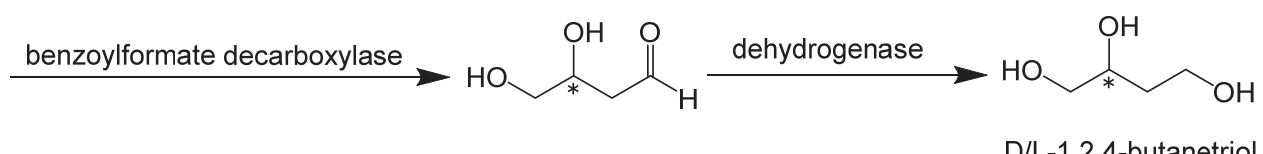

图4 四步酶促法合成BT ${ }^{[36]}$

迄今为止, 以木糖为底物的四步酶促反应法仍是最高效的BT生物合成途径 ${ }^{[41]}$ 。现研究人员将 $\mathrm{BT}$ 合成的 4 个关键酶基因在大肠杆菌中进行表达, 大大简化了流程工艺 ${ }^{[34]}$ 。但常用的大肠杆菌宿主存在 严重的碳代谢抑制(CCR), 限制了工程菌在混合糖下生长和合成BT ${ }^{[41]}$, 相比而言, 克雷伯氏菌 (Klebsiella pneumonia) 由于其生长速度更快, 对混合糖的利用率更高 ${ }^{[42,43,44]}$, 可以作为优化生物合 成途径的首选菌株。其他优化手段还包括：（1）敲除旁路基因，抑制分支反应; (2) 过表达关键酶; (3) 引入分子伴侣蛋白GroES等进行辅助折叠; (4) 采用反义RNA微调木糖代谢流以及弱化葡萄糖效 应。经过深入研究, 确定了四步反应的限速步骤为脱羧步骤, 并以优化该步的酶菌为重点, 使得 MDLC、KDCA、kivD等都较过去的催化酶而言有不同程度的提升 ${ }^{[45]}$ 。此外, 底物浓度、诱导温度、 诱导时机也都有一定的影响，通过篮选条件进行适度优化，提高BT生产效率。

\section{2 氧化偶氮化合物}

氧化偶氮化合物(Azoxy compound) 是另一类重要的含能材料, 这种含能材料中含有氧化偶氮键 (azoxy bond, 图5a), 可作为高爆炸药使用。氧化偶氮键的引入可以提高含能材料的密度, 并增加含 能材料的稳定性, 使材料的运输、使用更加安全 ${ }^{[32,46]}$ 。其中, 代表性的化合物为 $4,4^{\prime}$-二氨基-3,3'-氧 化偶氮呋咱(3,3'-diamino-4,4'-azoxyfurazan, DAOAF)和4,4'-二硝基-3,3'-氧化偶氮呋咱(3,3'-dinitro4,4'-azoxyfurazan, DNOAF) (图5b,5c) ${ }^{[46-48]}$ 。这两种化合物及其衍生物构成了主要的氧化偶氮基含 能材料 $[32,48-50]$ 。 
(a)<smiles>CN=NC</smiles>

(b)<smiles>Nc1nonc1N=Nc1nonc1N</smiles>

(c)<smiles>O=[N+]([O-])c1nonc1N=Nc1nonc1[N+](=O)[O-]</smiles>

图5（a) 氧化偶氮键；(b) DAOAF；(c) DNOAF

除了可用作含能材料外, 氧化偶氮化合物还有许多其他功能, 例如苯基氧化偶氮化合物可作为 液晶材料使用 ${ }^{[51]}$ 。然而, 用作高能材料或液晶材料的氧化偶氮化合物几乎完全依赖化学手段进行合 成, 经典的合成方法是, 使用 $O X O N E^{\mathrm{TM}}$ 氧化剂或者 $\mathrm{H}_{2} \mathrm{O}_{2}$ 氧化前体中的氨基, 并使之形成氧化偶氮键 [52-55]。近年来, 通过电化学催化将硝基苯衍生物氧化成氧化偶氮键的方法也层出不穷 ${ }^{[56,57]}$ 。

氧化偶氮化合物除了可以通过人工化学合成获取外, 部分天然产物中也含有氧化偶氮结构基 元 ${ }^{[58]}$, 这些天然产物具有诸如抗菌、抗癌等生物活性, 而它们的合成途径目前没有完全破解。浙江 大学李永泉团队 ${ }^{[59]}$ 在氧化偶氮化合物生物合成途径的研究中做出了重要的贡献, 他们对苯基氧化偶 氮类天然产物——氧化偶氮霉素的生物合成途径进行了系统性的研究, 并挖掘出了关键的金属酶。

氧化偶氮霉素 (Azoxymycins) 是李永泉课题组在恰塔努加链霉菌 L10 (Streptomyces Chattanoogensis L10)培养物中分离出的含有氧化偶氮键的新型天然产物, 分 $\mathrm{A} 、 \mathrm{~B}$ 和 $\mathrm{C}$ 三种, 通过多 维核磁、同位素取代、液相色谱、质谱与紫外光谱法确定了这三种天然产物的结构(图6)。鉴于氧化 偶氮霉素中含有苯多烯结构, 研究人员推定合成途径中可能存在与酮合成酶AsuC13/14同源的酶, 并 通过Native BLAST分析了 S. Chattanoogensis L10的基因组序列, 给出了推定的氧化偶氮霉素合成基 因簇(图7) ${ }^{[59] 。}$

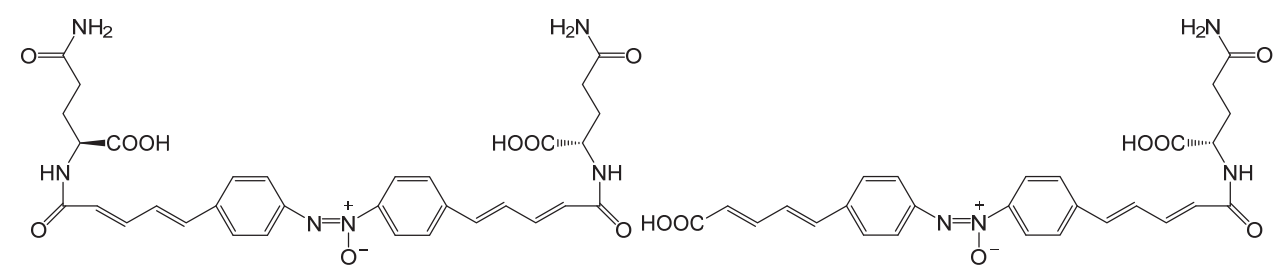

Azoxymaycin A

Azoxymaycin B

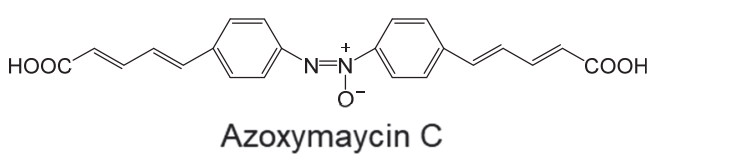

图6 氧化偶氮霉素A、B、C 的结构 ${ }^{[58]}$

Azo gene cluster

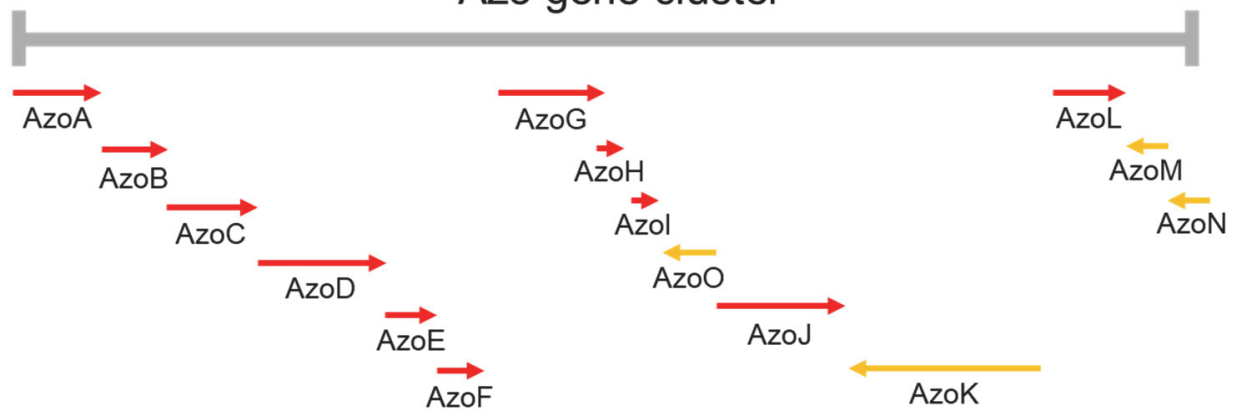

图7 氧化偶氮霉素合成基因簇 
深入研究发现, 在敲除 $a z o C$ 基因后, 培养物中会积累氧化偶氮霉素的苯胺前体产物, 因此研究 人员推定, AzoC酶是形成氧化偶氮键的关键酶。通过体外实验发现, AzoC酶可以催化包括氧化偶氮 霉素前体的一系列苯胺衍生物形成氧化偶氮键, 由此, AzoC酶的功能获得证实。通过生物信息学分 析与同源建模发现, $\mathrm{AzoC}$ 是一种与 $N$-加氧酶 $\mathrm{AurF}{ }^{[60]}$ 和 $\mathrm{CmlI}{ }^{[61]}$ 高度同源的非血红素双铁离子加氧

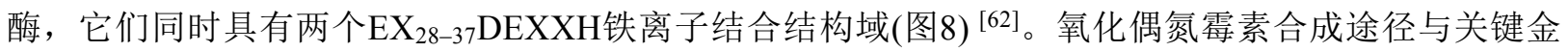
属酶的发现, 为其他氧化偶氮天然产物合成途径的解析提供了思路, 也为氧化偶氮含能材料的生物 合成奠定了基础。

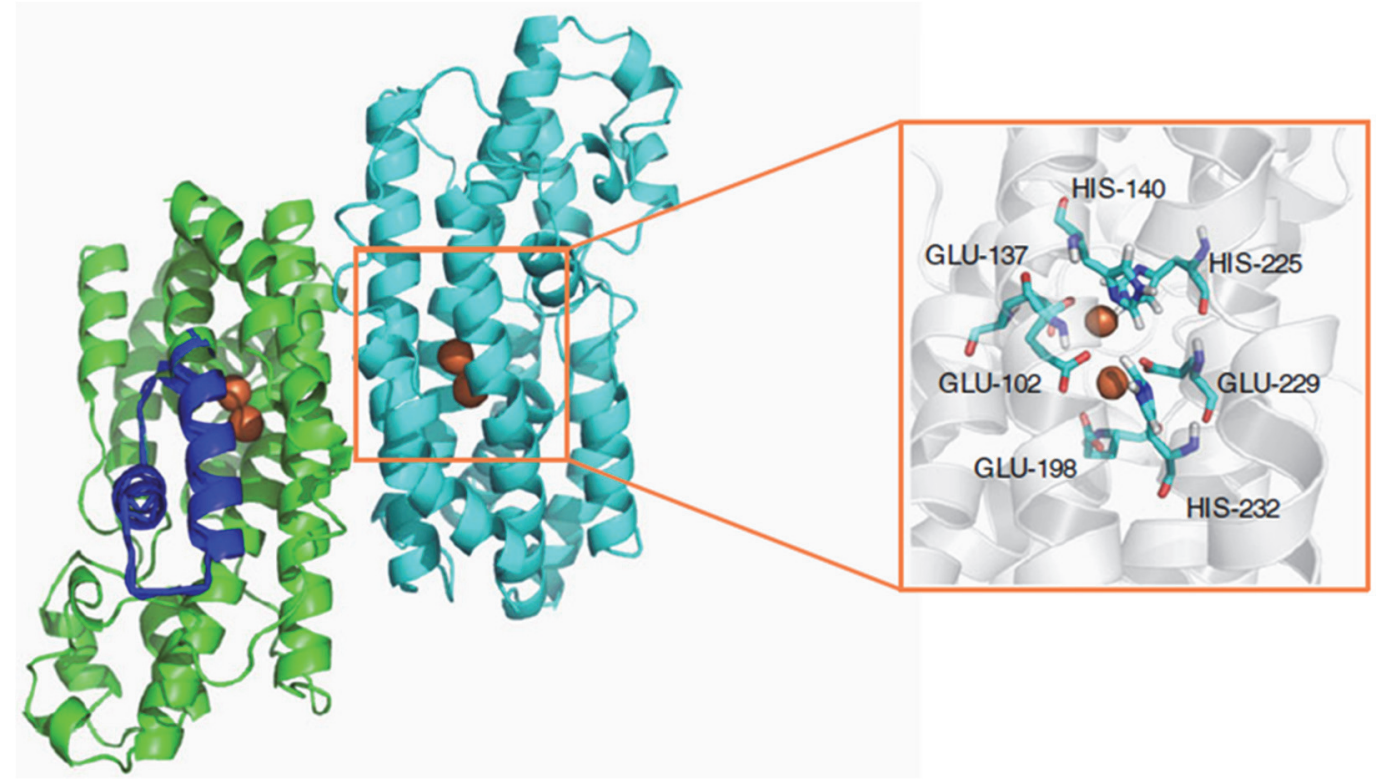

图8 AzoC同源建模结构, 示铁离子结合结构域[61]

生物合成含能材料仍有许多基础理论亟待突破, 目前含能材料的化学合成仍作为主要合成方式, 伴随着越来越多的生物合成途径的发现, 人们将在未来逐步实现以更清洁、更高效的生物方法合成 含能材料。

\section{3 天然高能物质的生物合成—ATP和PolyP}

与前述高能物质不同, 天然高能物质指的是含有高能键的物质, 如三磷酸腺苷ATP。此处 “高能 键” 并不是物理化学中所描述的键能高、强度大的化学键, 而是指在生物化学反应中能释放出大量 能量的化学键, 例如ATP中连接 $\gamma$-磷酸的高能磷酸二酯键。普通磷酸酯水解只能释放出 $8-16 \mathrm{~kJ} \cdot \mathrm{mol}^{-1}$ 的能量, 而ATP中 $\gamma$-磷酸水解时高能磷酸键的断裂可释放 $33-54 \mathrm{~kJ} \cdot \mathrm{mol}^{-1}$ 的能量 ${ }^{[63]}$ 。

多聚磷酸盐(Polyphosphate, PolyP)是一种由正磷酸盐残基与高能磷氧键组成的结构最为紧凑的 生物大分子, 是一种广泛存在于生物体胞内的、进化上高度保守的储能高分子材料。根据多聚磷酸 盐对各类生物生命活动的重要意义 ${ }^{[64-66]}$, 可将其分为两大类, 即焦磷酸盐和大分子聚磷酸盐 ${ }^{[65]}$ 。

一个大分子的聚磷酸盐中含有三个甚至几百个磷酸残基, 其功能和代谢方式也与焦磷酸盐有 着明显的区别 ${ }^{[65]}$ 。1990年, 诺贝尔化学奖得主Kornberg团队 ${ }^{[67,68]}$ 首次发现并报道了细菌中的PolyP 合成酶一一多聚磷酸盐激酶(Polyphosphate kinase, PPK), 这一发现使从基因水平操纵生物体内 PolyP 的合成成为现实。该团队继而开发了多种关于PolyP的检测和定量方法 ${ }^{[69,70]}$, 为系统性研究PolyP在 生物生理活动中的作用做出了巨大贡献。2005年, PPK的结构首次得到确定, 通过X射线晶体学方法 可知, PPK是一种以 $\mathrm{Mg}^{2+}$ 为辅因子的二聚体金属酶(图9) ${ }^{[71]}$ 。 


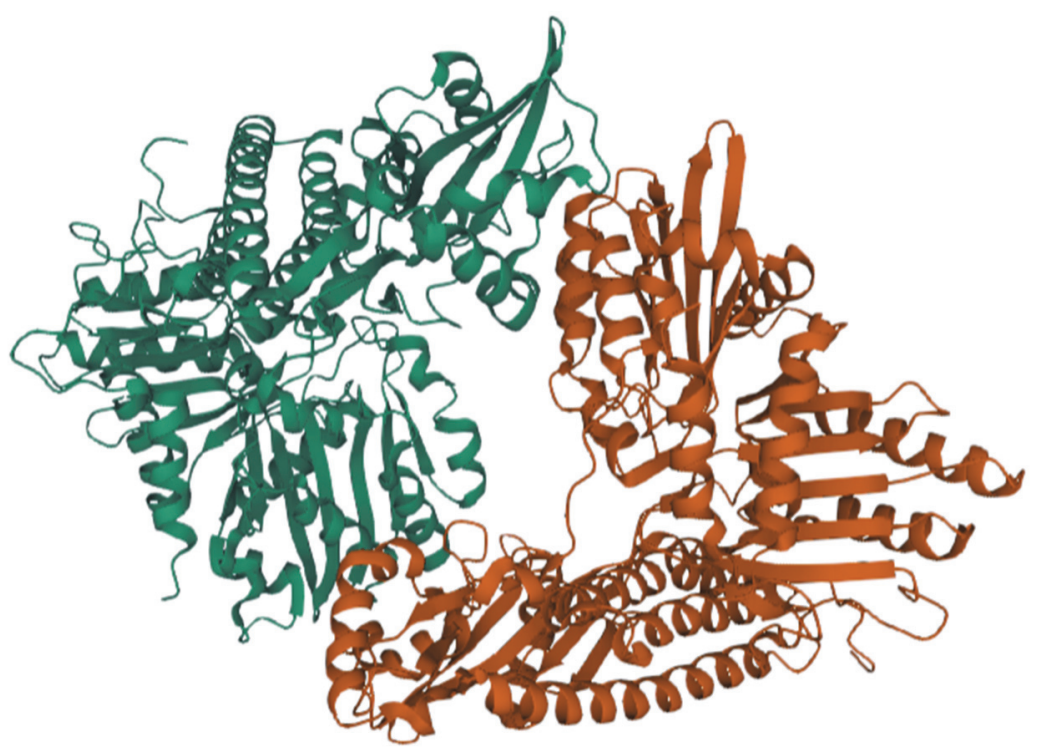

图9 PPK晶体结构(PDBID: 1XDO) ${ }^{[70]}$

当下对聚磷微生物的了解大部分来自污水处理工程中强化生物除磷系统(Enhanced biological phosphorus removal, EBPR)的广泛研究 ${ }^{[72]}$, 这为PolyP的生物合成路径提供了灵感。南京大学杨柳燕 团队利用合成生物学技术, 采用导入单个ppk 1 基因过表达中拷贝质粒的策略, 构建了一系列工程菌, 可通过富集富磷水中的磷元素来制备高聚合度的PolyP, 磷元素回收率大于 $95 \%{ }^{[73]}$ 。此外, 近期也有 报道称可利用酿酒酵母合成水溶性的食品级的PolyP ${ }^{[74-76]}$, 但关于 vtc1-vtc4基因产物在PolyP合成中 的酶活性尚在探索当中 ${ }^{[77]}$, 幸运的是, VTC复合体的结构已经解析, VTC-PolyP复合体的结构也已 经获取(图 10) ${ }^{[78], ~}$ 这对理解PolyP的生物功能、促进PolyP合成工业的发展具有重大意义。

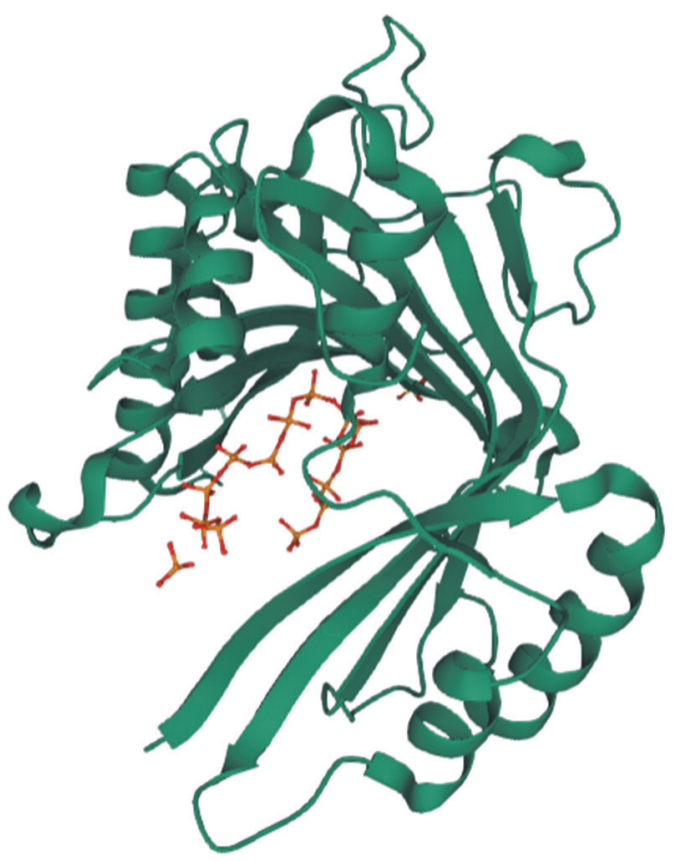

图10ＶTC-PolyP复合体结构(PDBID: 3G3Q) ${ }^{[77]}$ 
与化学合成的低聚合度多聚磷酸盐(链长 $<20 \mathrm{Pi}$ )不同, 生物合成的多聚磷酸盐聚合度更高 $(20 \mathrm{Pi}<$ 链长 $<700 \mathrm{Pi}$ ), 单位负电荷密度更高 ${ }^{[73,77]}$, 更具功能性与特征性。南京大学赵劲与魏炜团队 ${ }^{[79]}$ 发 现, PolyP可与细胞中的强正电蛋白质结合并引发相分离现象, 意味着PolyP具有未知的生物调控功 能。现在科学家已通过实验表明: PolyP能够与带正电荷的聚合物(如: 海藻酸、透明质酸)、无机阳 离子(如: $\mathrm{Ca}^{2+} 、 \mathrm{Mg}^{2+} 、 \mathrm{Sr}^{2+}$ )或基本有机成分(如: 氨基酸、多胺、多肽、蛋白质)相互作用, 加工成水 凝胶或纳米颗粒, 用于人工骨修复材料和其他生物医学应用 ${ }^{[80,81] 。}$

以聚磷酸盐为代表的天然高能产物, 其生物功能仍有许多未解之谜。天然高能物质的生物合成 的发展, 将大大降低这些物质的获取难度与生产成本, 为破解这些科学问题提供物质基础, 同时也 将为人们提供一批新型的天然高分子材料, 助力人类重大疾病的攻克。

\section{4 展望}

进入 21 世纪以来, 生物合成技术虽然得到了长足的发展, 但距离以生物合成替代化学合成工业 还有着很长的路要走, 在高能物质生物合成领域, 不少生物合成途径仍等待破解, 工业化生产也亟 待突破。目前的瓶颈在于: 生物合成能替代的化学合成技术太少, 不足以应对高能化学物质的需求; 生物合成成本还较高, 需要发展高效的工艺; 由于合成生物学和能源物质结合刚刚起步, 很多应用 场景还需要拓展。这些挑战需要我们在未来将基础研究与应用发展并重, 并通过生物合成途径的基 础研究来促进工业转化，用更高效的酶和更简捷的路线来实现更高的产能。

随着人们对环境友好型产业的期待, 以及国家对绿色生物合成技术发展的重视, 在可预见的未 来, 生物合成高能物质必将成为中国乃至世界范围内能源结构的重要组成部分, 对人类未来能源获 取和转化方式产生深刻影响。

\section{参考文献}

[1] 关于碳达峰、碳中和, 总书记这样说. [2021-09-17]. http://www.qstheory.cn/zhuanqu/2021-09/17/c_1127873054.htm

[2] 政府工作报告. [2021-09-17]. http://www.gov.cn/guowuyuan/zfgzbg.htm

[3] 谭天伟, 陈必强, 张会丽, 崔子恒. 化工进展, 2021, 40 (3), 1137.

[4] 杨荣武. 生物化学原理. 第3版. 北京: 高等教育出版社, 2018.

[5] Keasling, J. D. ACS Chem. Biol. 2008, 3 (1), 64.

[6] 王凯, 刘子鹤, 陈必强, 王萌, 张洋, 毕浩然, 周雅莉, 霍奕影, 谭天伟. 合成生物学, 2020, 1 (1), 60 .

[7] 史硕博, 孟琼宇, 乔玮博, 赵惠民. 合成生物学, 2020, l (1), 44.

[8] 徐硕, 余碧䒯. 北京理工大学学报(社会科学版), 2021, 23 (6), 1.

[9] 白羽. 莱茵衣藻[FeFe]氢化酶HydA1体内区域性成熟过程的研究[博士学位论文]. 厦门: 厦门大学, 2018.

[10] Shima, S.; Pilak, O.; Vogt, S.; Schick, M.; Stagni, M. S.; Meyer-Klaucke, W.; Warkentin, E.; Thauer, R. K.; Ermler, U. Science 2008, 321 (5888), 572 .

[11] Hiromoto, T.; Warkentin, E.; Moll, J.; Ermler, U.; Shima, S. Angew. Chem. Int. Ed. 2009, 48 (35), 6457.

[12] Corr, M. J.; Murphy, J. A. Chem. Soc. Rev. 2011, 40 (5), 2279.

[13] Shima, S.; Chen, D.; Xu, T.; Wodrich, M. D.; Fujishiro, T.; Schultz, K. M.; Kahnt, J.; Ataka, K.; Hu, X. Nat. Chem. 2015,7 (12), 995.

[14] Nicolet, Y.; Piras, C.; Legrand, P.; Hatchikian, C. E.; Fontecilla-Camps, J. C. Structure (London) 1999, 7 (1), 13.

[15] Mulder, D. W.; Shepard, E. M.; Meuser, J. E.; Joshi, N.; King, P. W.; Posewitz, M. C.; Broderick, J. B.; Peters, J. W. Structure. 2011,19 (8), 1038.

[16] Senger, M.; Eichmann, V.; Laun, K.; Duan, J.; Wittkamp, F.; Knör, G.; Apfel, U.; Happe, T.; Winkler, M.; Heberle, J.; et al. J. Am. Chem. Soc. 2019, $141(43), 17394$.

[17] Volbeda, A.; Garcin, E.; Piras, C.; de Lacey, A. L.; Fernandez, V. M.; Hatchikian, E. C.; Frey, M.; Fontecilla-Camps, J. C. J. Am. Chem. Soc. 1996, $118(51), 12989$. 
[18] Mcdowall, J. S.; Murphy, B. J.; Haumann, M.; Palmer, T.; Armstrong, F. A.; Sargent, F. Proc. Natl. Acad. Sci. USA 2014, 111 (38), E3948.

[19] Mcdowall, J. S.; Hjersing, M. C.; Palmer, T.; Sargent, F. FEBS Lett. 2015, 589 (20PartB), 3141.

[20] Cestellos-Blanco, S.; Zhang, H.; Kim, J. M.; Shen, Y.; Yang, P. Nat. Catal. 2020, 3 (3), 245.

[21] Fujishima, A.; Honda, K. Nature 1972, 238 (5358), 37.

[22] Honda, Y.; Hagiwara, H.; Ida, S.; Ishihara, T. Angew. Chem. Int. Ed. 2016, 55 (28), 8045.

[23] Xiong, W.; Zhao, X.; Zhu, G.; Shao, C.; Li, Y.; Ma, W.; Xu, X.; Tang, R. Angew. Chem. Int. Ed. 2015, 54 (41), 11961.

[24] Wei, W.; Sun, P.; Li, Z.; Song, K.; Su, W.; Wang, B.; Liu, Y.; Zhao, J. Sci. Adv. 2018, 4 (2), 9253.

[25] Chen, J.; Li, J.; Li, Q.; Wang, S.; Wang, L.; Liu, H.; Fan, C. Energy Environ. Sci. 2020, 13 (7), 2064.

[26] Sokol, K. P.; Robinson, W. E.; Warnan, J.; Kornienko, N.; Nowaczyk, M. M.; Ruff, A.; Zhang, J. Z.; Reisner, E. Nat. Energy 2018, 3 (11), 944.

[27] Zhang, L.; Morello, G.; Carr, S. B.; Armstrong, F. A. J. Am. Chem. Soc. 2020, 142 (29), 12699.

[28] Pan, H. J.; Hu, X. Angew. Chem. Int. Ed. 2020, 59 (12), 4942.

[29] Wang, X. Z.; Meng, S. L.; Xiao, H.; Feng, K.; Wang, Y.; Jian, J. X.; Li, X. B.; Tung, C. H.; Wu, L. Z. Angew. Chem. Int. Ed. 2020, 59 (42), 18400.

[30] Evans, R. M.; Krahn, N.; Murphy, B. J.; Lee, H.; Armstrong, F. A.; Söll, D. Proc. Natl. Acad. Sci. USA 2021, 118 (13), e2100921118.

[31] 吴雄辉. 偶氮及氧化偶氮呋咱类高能化合物的合成与性能预测 [硕士学位论文]. 南京: 南京理工大学, 2016.

[32] Badgujar, D. M.; Talawar, M. B.; Asthana, S. N.; Mahulikar, P. P. J. Hazard. Mater. 2008, 151 (2-3), 289.

[33] Kohli, K.; Prajapati, R.; Sharma, B. Energies 2019, 12 (2), 223.

[34] Valdehuesa, K. N. G.; Liu, H.; Ramos, K. R. M.; Park, S. J.; Nisola, G. M.; Lee, W.; Chung, W. Process Biochem. 2014,49 (1), 25.

[35] Mainguet, S. E.; Liao, J. C. Biotechnol. J. 2010, 5 (12), 1297.

[36] Niu, W.; Molefe, M. N.; Frost, J.W. J. Am. Chem. Soc. 2003, 125 (43), 12998.

[37] Yukawa, T.; Bamba, T.; Guirimand, G.; Matsuda, M.; Hasunuma, T.; Kondo, A. Biotechnol. Bioeng. 2021, 118 (1), 175.

[38] Zhao, M.; Shi, D.; Lu, X.; Zong, H.; Zhuge, B. Bioresour. Technol. 2019, 282, 433.

[39] Hanessian, S.; Ugolini, A.; Dubé, D.; Glamyan, A. Can. J. Chem. 1984, 62 (11), 2146.

[40] Tandon, V. K.; Van Leusen, A. M.; Wynberg, H. J. Org. Chem. 1983, 48 (16), 2767.

[41] 李玉石, 刘郁青, 杨程雨, 陆信曜. 微生物学通报, 2020, 47 (8), 2505.

[42] 马鹏飞, 蒙坚, 周静. 高海军. 化工学报, 2015, 66 (7), 2620.

[43] 张根林, 邓辉, 鲁建江, 孙守玲, 李春. 过程工程学报, 2009, 9 (6), 1174.

[44] 陈柳霓, 李笑寒, 田平芳. 生物加工过程, 2017, 15 (4), 34.

[45] Yim, H.; Haselbeck, R.; Niu, W.; Pujol-Baxley, C.; Burgard, A.; Boldt, J.; Khandurina, J.; Trawick, J. D.; Osterhout, R. E.; Stephen, R.; et al. Nat. Chem. Biol. 2011, 7 (7), 445 .

[46] Zhang, J.; Shreeve, J. N. M. J. Phys. Chem. C 2015, 119 (23), 12887.

[47] 王小旭. 百克级高纯DAOAF的合成技术及工艺研究[硕士学位论文]. 绵阳: 西南科技大学, 2018.

[48] Zhang, J.; Shreeve, J. N. M. J. Am. Chem. Soc. 2014, 136 (11), 4437.

[49] 吴雄辉. 偶氮及氧化偶氮呋咱类高能化合物的合成与性能预测[硕士学位论文]. 南京: 南京理工大学, 2016.

[50] Liu, Y.; Zhang, J.; Wang, K.; Li, J.; Zhang, Q.; Shreeve, J. N. M. Angew. Chem. Int. Ed. 2016, 55 (38), 11548.

[51] Iamsaard, S.; Aßhoff, S. J.; Matt, B.; Kudernac, T.; Cornelissen, J. J.; Fletcher, S. P.; Katsonis, N. Nat. Chem. 2014,6 (3), 229.

[52] Francois, E. G.; Chavez, D. E.; Sandstrom, M. M. Prop. Exp. Pyrotech. 2010, 35, 529.

[53] Wu, X.; Chu, Y.; Zheng, C.; Wang, T.; Lei, W.; Wang, F.; Xia, M. Chem. Heterocycl. Compd. 2015,51 (8), 760.

[54] Han, S.; Cheng, Y.; Liu, S.; Tao, C.; Wang, A.; Wei, W.; Yu, H.; Wei, Y. Angew. Chem. Int. Ed. 2021, 60 (12), 6382.

[55] Gulyaev, D. A.; Klenov, M. S.; Churakov, A. M.; Strelenko, Y. A.; Fedyanin, I. V.; Lempert, D. B.; Kosareva, E. K.; Kon'Kova, T. S.; Matyushin, Y. N.; Tartakovsky, V. A. RSC Adv. 2021, 11 (39), 24013.

[56] Chong, X.; Liu, C.; Huang, Y.; Huang, C.; Zhang, B. Natl. Sci. Rev. 2020, 7 (2), 285.

[57] Sadatnabi, A.; Mohamadighader, N.; Nematollahi, D. Org. Lett. 2021, 23 (16), 6488.

[58] Katsuyama, Y.; Matsuda, K. Curr. Opin. Chem. Biol. 2020, 59, 62. 
[59] Guo, Y.; Li, H.; Zhou, Z.; Mao, X.; Tang, Y.; Chen, X.; Jiang, X.; Liu, Y.; Jiang, H.; Li, Y. Org. Lett. 2015,17 (24), 6114.

[60] Zocher, G.; Winkler, R.; Hertweck, C.; Schulz, G. E. J. Mol. Biol. 2007, 373 (1), 65.

[61] Lu, H.; Chanco, E.; Zhao, H. Tetrahedron 2012, 68 (37), 7651.

[62] Guo, Y. Y.; Li, Z. H.; Xia, T. Y.; Du, Y. L.; Mao, X. M.; Li, Y. Q. Nat. Commun. 2019, 10 (1), 1.

[63] 汪小兰. 有机化学. 第5版. 北京: 高等教育出版社, 2017.

[64] Brown, M. R.W.; Kornberg, A. Proc. Natl. Acad. Sci. USA 2004, 101 (46), 16085.

[65] Kulaev, I. S.; Vagabov, V. M.; Kulakovskaya, T. V. The Biochemistry of Inorganic Polyphosphates; John Wiley \& Sons: New Jersey, USA, 2004.

[66] Moradali, M. F.; Rehm, B. Nat. Rev. Microbiol. 2020, 18 (4), 195.

[67] Ahn, K.; Kornberg, A. J. Biol. Chem. 1990, 265 (20), 11734.

[68] Zhang, H.; Ishige, K.; Kornberg, A. Proc. Natl. Acad. Sci. USA 2003, 99 (26), 16678.

[69] Ault-Riché, D.; Fraley, C. D.; Tzeng, C. M.; Kornberg, A. J. Bacteriol. 1998, 180 (7), 1841.

[70] Rao, N. N.; Liu, S.; Kornberg, A. J. Bacteriol. 1998, 180 (8), 2186.

[71] Zhu, Y.; Huang, W.; Lee, S. S. K.; Xu, W. EMBO Rep. 2005, 6 (7), 681.

[72] Akbari, A.; Wang, Z.; He, P.; Wang, D.; Lee, J.; Han, I. L.; Li, G.; Gu, A. Z. Microb. Biotechnol. 2021,14 (1), 82.

[73] Wang, X.; Wang, X.; Hui, K.; Wei, W.; Zhang, W.; Miao, A.; Xiao, L.; Yang, L. Environ. Sci. Technol. 2018,52 (1), 214.

[74] Christ, J. J.; Blank, L. M. FEMS Yeast. Res. 2019, 19 (3), foz011.

[75] Christ, J. J.; Smith, S. A.; Willbold, S.; Morrissey, J. H.; Blank, L. M. Biotechnol. Bioeng. 2020, 117 (7), 2089.

[76] Kulakovskaya, T.V.; Andreeva, N. A.; Ledova, L. A.; Ryazanova, L. P.; Trilisenko, L.V.; Eldarov, M. A. Biochem. Moscow 2021, 86, S96.

[77] Rao, N. N.; Gomez-Garcia, M. R.; Kornberg, A. Annu. Rev. Biochem. 2009, 78, 605.

[78] Hothorn, M.; Neumann, H.; Lenherr, E. D.; Wehner, M.; Rybin, V.; Hassa, P. O.; Uttenweiler, A.; Reinhardt, M.; Schmidt, A.; Seiler, J.; et al. Science 2009, 324 (5926), 513.

[79] Wang, X.; Shi, C.; Mo, J.; Xu, Y.; Wei, W.; Zhao, J. Angew. Chem. Int. Ed. 2020, 59 (7), 2679.

[80] Wu, A. T. H.; Aoki, T.; Sakoda, M.; Ohta, S.; Ichimura, S.; Ito, T.; Ushida, T.; Furukawa, K. S. Biomacromolecules 2015,16 (1), 166.

[81] Chen, D.; Zhou, X.; Chang, L.; Wang, Y.; Li, W.; Qin, J. Biomacromolecules 2021, 22 (5), 2272. 